\title{
A Global Perspective In Controlling Hepatitis B Virus Through Vaccination: A Review With A Focus On Indonesia
}

\author{
Dewi Ratna Sulistina ${ }^{\mathrm{a}}$, Maria I. Lusida ${ }^{\mathrm{b}}$, Santi Martinic ${ }^{\mathrm{c}}$, Budi Prasetyo ${ }^{\mathrm{d}}$, \\ ${ }^{a}$ Doctoral Study Program, Faculty of Public Health, Universitas Airlangga, Surabaya, East Java, Indonesia, \\ dewiratnasulistina@gmail.com, dewi.ratna.ua@gmail.com \\ ${ }^{\mathrm{b}}$ Institute of Tropical Disease, Universitas Airlangga, Surabaya, Indonesia; Epidemiology Department, \\ ${ }^{\mathrm{c}}$ Faculty of Public Health, Universitas Airlangga, Surabaya, East Java, Indonesia; \\ ${ }^{\mathrm{d}}$ Department of Social Obstetrics and Gynecology, Faculty of Medicine, Universitas Airlangga
}

\begin{abstract}
Even though the universal hepatitis B vaccination program has been conducted in Indonesia since 1997, but until 2018, it has not fulfilled the expected target and tends to experience a decreasing trend. The result of this study was expected to contribute to the government in formulating the best approach to succeed the hepatitis B vaccination program in Indonesia and other countries in the world in the future.

The survey of empirical studies and the analysis of articles related to the global perspective in controlling hepatitis B virus through vaccination: a review with a focus on Indonesia.

The global prevalence of HBsAg in 2016 was 3.9\%. The prevalence of HBsAg in Indonesia in 2016 showed that the population of adults and pregnant women were at risk of being infected with hepatitis B compared to pre-school students and other students. Giving hepatitis B vaccine recombinant in adolescents aged 15-18 years was proven to help give a high immunity and safe along with the decrease in anti-HBs in children after universal vaccination for newborn babies. According to the national immunization policy in Indonesia, the hepatitis B birth dose vaccine should be given within 7 days after the birth and followed by the combination of three vaccines, including diphtheria, pertussis, tetanus, and hepatitis B in 2, 3, and 4 months (DPT-HB3). Both hexavalent (DTaPHB-Hib-IPV) and pentavalent (DTwP-HB-Hib) vaccines were effective in stimulating anti-HBs $\geq 10 \mathrm{mIU} / \mathrm{ml}$, and therefore, both of them can be used as a single formula booster at the age of 18 months for simplifying the vaccination. A study identified two dominant genotypes (B and C) among adults in Indonesia. Furthermore, four zones of HBV subtype shared patterns identified in Indonesia: adw, ayw, adr, and mix. The development of vaccines and the detection of mutation passing the diagnostics in the antigenic epitopes in HBsAg will be beneficial for the authority of public health. Further, the diversity of nucleotide levels and amino acids as well as the difference in the proportion of $\mathrm{dN} / \mathrm{dS}$ in PreS1, PreS2, and HBsAg showed a tendency of selective pressure of non-active status to advanced liver disease. A new policy related to School-Based Vaccination needs to be introduced in Indonesia with a regular medical check-up for vaccination status when registering the school or during elementary school. With an increased number of new vaccines and the dose of vaccine boosters for children targeting school-age students, SBV implementation, and the inspection of students' vaccination records at school can help increase the vaccination coverage.
\end{abstract}

Keywords: Perspective, Controlling Hepatitis B, Vaccination

\section{Introduction}

The Asia-Pacific region is home to more than half of the global population and contributes $62.6 \%$ of global mortality due to liver disease in 2015 . Around $54.3 \%$ of global mortality is caused by cirrhosis. $72.7 \%$ of global mortality is caused by hepatocellular carcinoma, and more than two-thirds of the global burden of acute viral hepatitis happened in this region in 2015 (1). South Asia region, including Indonesia, is the home for nearly 39 million people with chronic HBV and it is estimated around 10 million people with HCV. It is estimated around 410,000 people were died (78\% of the total population) annually due to hepatitis virus with a complication of chronic HBV and HCV (2).

Immunization is one of the most cost-saving health interventions to prevent diseases, disorders, and mortality related to infectious diseases. Indonesia adopted a universal hepatitis B vaccination program for babies in 1997. The plasma-derived vaccine had been produced until 1997; right after that, it was replaced with a hepatitis B vaccine recombinant. In 1999, Indonesia introduced an innovative policy by using a single-use syringe from a stable hepatitis B vaccine, even outside the cold chain. This approach has increased the coverage of the hepatitis B vaccine and decreased the rate of hepatitis B carriers. In 2000, Indonesia introduced a program for using an instrument for the birth dose in seven provinces. In 2003, this program was extended to target 5 million births in Indonesia. The outcome of the national hepatitis B immunization program is the HBsAg prevalence of less than $1 \%$ after five years of the regular immunization started (3).

Even though the universal hepatitis B universal vaccination program has been conducted since 1997, until 2018, it did not reach the expected target and tended to have a declining trend. Based on the result from the Basic Health Research in 2018, the proportion of DPT-HB 3/DPT-HB-HiB 3 immunization 
in children aged 12-23 months from 2013 to 2018 showed a declining trend of $15.3 \%$ (from $76.6 \%$ to $61.3 \%)(4)$.

\section{Methods}

This study was conducted based on the survey of general empirical studies on the global perspective in controlling hepatitis B virus through vaccination: a review with a focus on Indonesia. In this study, the researcher conducted a general investigation entitled "A Global Perspective in Controlling Hepatitis B Virus through Vaccination". From this investigation, the researcher found many abstracts of articles that had been thoroughly read to determine which article that would be included in this review. After thoroughly being read, the majority of the collected articles had the following approach: case study and qualitative analysis. In this paper, the researcher directly focused on empirical studies and keywords related to a global perspective in controlling the hepatitis B virus through vaccination.

\section{Results and Analysis}

The global prevalence of HBsAg in 2016 was 3.9\%. From this infection, around 29 million people (10\%) were diagnosed, and only 4.8 million (5\%) out of 94 million people fulfilling the requirements for having treatment received anti-virus therapy. Around 1.8 (1.6-2.2) million infections occurred in children aged 5 years with a prevalence of $1.4 \%(1.2-1.6)$. It was estimated around $87 \%$ of the babies had received three doses of HBV vaccination in their first year of life; $46 \%$ of them had received the birth dose vaccination on time, and $13 \%$ had received the hepatitis B immunoglobulin along with a complete vaccination regimen. Less than $1 \%$ of mothers with a high viral load had received antivirus therapy to minimize the transmission from mothers to children (5).

In Taiwan, one of the countries with the highest hepatitis B-endemic rate that successfully implemented the universal vaccination program, the prevalence of HBsAg decreased from $9.8 \%$ in 1984 , the year when the universal vaccination of newborn babies started, to $0.7 \%$ in 1999 , or after the following 15 years. The hepatitis B and immunoglobulin (HBIG) vaccine is the most effective method (89-98\%) to prevent perinatal transmission if it was given 12-24 hours after birth. Reflecting on the success in implementing the universal vaccination program, Indonesia has adopted the universal hepatitis B vaccination program for newborn babies since 1997. After adopting it for 15 years, in 2012, the prevalence of HBsAg in preschool children and school-age children was from $2.1 \%$ to $4.2 \%$ and $0 \%$ to $5.9 \%$. This percentage is lower than the previous study in adult populations (from 5 to 10\%) and pregnant women $(5.2 \%)$ in Indonesia. In this study, the prevalence of seropositive for anti-HBs in preschool-age children was higher (61.4-65.8\%) than that of school-age children (20.9-40.4\%) (3). The prevalence of hepatitis B infection in children born during the national immunization program era in West Kotawaringin Regency (Central Kalimantan) and Kupang (East Nusa Tenggara) was still quite high at $3.1 \%$ and $5.1 \%$ consecutively with HBV genotypes B and C. That being the case, the proportion of HBV carriers in both of these regions was still above the national target or less than $2 \%$ (6). The overall prevalence of hepatitis B infection in children born during the national immunization program era in Sumenep, Madura, was 6 (5.88\%) out of 102 samples positive for $\mathrm{HBsAg}, 51$ (50.00\%) out of 102 samples positive for anti-HBs, and $49(48.04 \%)$ out of 102 samples positive for anti-HBc. The proportion of HBsAg in Sumenep, Madura, was still high, even after the universal vaccination program was implemented; the protective antibody against the surface antigen of hepatitis $B$ was adequate, but there was prejudice for occult infections (hidden infections) of hepatitis $\mathrm{B}(\mathrm{OBI})$ (7). This prevalence showed that the population of adults and pregnant women were at risk of being infected with hepatitis B more than preschool-age and school-age children. A previous study conducted on children vaccinated in Taiwan showed that the positivity of anti-HBs for children aged 2 years was around $93-97 \%$. The antibody titer was then gradually decreased to less than 10 $\mathrm{mIU} / \mathrm{mL}$ in 10-15 years. The result of this study might reflect the similar decrease in the level of anti-HBs; however, the lower coverage of immunization in Indonesia during the early years of its implementation (before 2003) might also contribute to this result. It is in line with a study conducted by Efendi that the prevalence of full immunization status for the age group of 12-23 months in Indonesia was $37.4 \%$. The highest coverage of immunization was BCG (86.9\%) and the lowest was hepatitis B (46.2\%) (8). It is also in line with a study conducted by Fadlyana that giving hepatitis B vaccine recombinant in adolescents aged around 15-18 years in three doses $(1.0 \mathrm{ml}=20 \mu \mathrm{g}$ of $\mathrm{HbsAg})$ was proven to give a strong and safe immunity to be given to healthy adolescents. A study on giving hepatitis B vaccine recombinant in adolescents aged 15-18 years helped give a strong and safe immune along with the decrease in anti-HBs of less than 10 $\mathrm{mIU} / \mathrm{mL}$ in children after universal vaccination for newborn babies (9).

The factors associated with the coverage of immunization completeness and the coverage of hepatitis B birth dose vaccine are as follows: many people in isolated areas in Indonesia deal with 
difficulties to reach health centers due to geographic isolation. Further, hepatitis B screening for all pregnant women is not performed as the national program in Indonesia, and the majority of newborn babies born from women with hepatitis B will, therefore, never receive HBIG. If mothers are infected and transmit the virus before the babies are vaccinated and there is no HBIG to be given at the same time in 24 hours after the birth, the vaccination will not protect the babies. According to the data from the local Office of Health, the coverage of DPT-HB3 in Central Kalimantan, West Timor, and West Papua is quite high (73.9-94.1\%). In contrast, the coverage of hepatitis B birth dose vaccine in those areas is less than $50 \%$. Many people in Indonesia who live far from the health care centers prefer giving birth at home and getting a traditional birth attendant to give birth to babies. These facts can delay the schedule of hepatitis B birth dose vaccination. This study showed the current geographic distribution pattern from the genotype and subtype of HBV among children in 15 years after the implementation of the universal vaccination program (3); mothers with a firstborn child have 2.84 times higher possibility to vaccinate their babies fully than mothers with $\geq 6$ children. Mothers with the lowest economic level, giving birth at non-health facility centers, attending antenatal care less than four times tended to have less possibility to vaccinate their children fully. Meanwhile, mothers giving birth assisted by professional birth attendants are significantly correlated with the status of complete immunization of their children. An accurate strategy needs to be devised by targeting a group with a high risk, including a cultural approach, to solve the completion of immunization and the increase in accessibility in many provinces in this country (8).

According to the policy on national immunization in Indonesia, the hepatitis B birth dose vaccine should be given in 7 days after the birth and followed by three combination vaccines, including diphtheria, pertussis, tetanus, and hepatitis B in 2, 3, and 4 months (DPT-HB3) (3). A study in 2 groups of newborn babies (previously the mothers received the following vaccines: tetanus-diphtheria-acellular pertussis (Tdap)) then they were randomly vaccinated with pentavalent and hexavalent at the age of 2, 4, 6, and 18 months after HB monovalent vaccine at birth. The blood sample was taken after born, a month after primary immunization series (month 7), pre-booster (month 18), a month of post-booster (month 19), and six months after booster (month 24). The third group of newborn babies whose mothers did not receive Tdap was vaccinated with DTwP-HB-Hib (EPI pentavalent group). The levels of $\mathrm{HBs} \mathrm{Ag}$, anti-HBc, and anti-HBs were evaluated using the automatic Chemiluminescent Microparticle Immunoassay. The levels of anti-HBs $\geq 10 \mathrm{mIU} / \mathrm{ml}$ in newborn babies were $99.2 \%$ (hexavalent group), 99.2\% (pentavalent group), and $98.5 \%$ (EPI pentavalent group), after four doses of immunization (at the age of $0,2,4$, and 6 months). A month after an additional dose given at the age of 18 months, the levels of anti-HBs $\geq 10 \mathrm{mIU} / \mathrm{ml}$ observed in newborn babies were $100 \%$ (hexavalent group), $99.2 \%$ (pentavalent group), and $93.8 \%$ (EPI pentavalent group). At the age of 24 months, the babies reaching the higher level of anti-HBs $\geq 10 \mathrm{mIU} / \mathrm{ml}$ were found in the hexavalent group $(98.3 \%)$ compared to the pentavalent group (86.5\%). Both vaccines, hexavalent (DTaP-HB-Hib-IPV) and pentavalent (DTwP-HB-Hib), are effective in stimulating the level of anti-HBs $\geq 10 \mathrm{mIU} / \mathrm{ml}$, and therefore, both of them can be used as the single formula booster at the age of 18 months for simplifying the vaccination under the Extended Program on Immunization in Thailand (10).

A study on the immunogenicity response of newborn babies after getting the four doses of bivalent oral polio vaccine (bOPV; Bio Farma), given along with DTwP-Hb-Hib (Pentabio) and one dose of inactivated poliovirus vaccine (IPV) was conducted in the fourth visit. A total of 143 newborn babies fulfilled the listed inclusion criteria and completed the study. The subject received the first dose of bOPV after being born. On day 60, 90, and 120, the bOPV was given along with Pentabio. On day 120, one dose of IPV was also given. The serum sample for serological analysis was collected before the first dose of bOPV (day 0), before the second dose of bOPV (day 60), and 30 days after the last dose of bOPV. Besides, the duration and the correlation of each damaging the vaccination experiment were evaluated. The seroprotection rates after the fourth dose of bOPV were $100 \%, 91.6 \%$, and $99.3 \%$ for poliovirus $\mathrm{P} 1, \mathrm{P} 2$, and $\mathrm{P} 3$ respectively. The seroconversion rates after the fourth dose of OPV were $100.0 \%, 93.3 \%$, and $100 \%$ for poliovirus P1, P2, and P3 respectively. There was no severe side effect, and the systemic reaction is generally mild for a period of 1-28 days of post-vaccination. Collectively, the finding showed that giving bOPV along with Pentabio and one dose of IPV in the fourth visit was immunogenic and well-tolerated without any serious problem related to the side effects of the vaccine (11).

A previous study identified four genotypes (A, B, C, and D) in adult people in Indonesia; nevertheless, genotypes B and C showed a dominant contribution. Further, the four zones of HBV subtype shared the pattern identified in Indonesia: adw, ayw, adr, and mix (3). A phylogenetic study conducted on 319 patients infected with HBV to figure out the genotypes and subgenotypes of isolates in Saudi Arabia showed a different clinical manifestation, including active and inactive chronic carriers as well as patients with cirrhosis and hepatocellular carcinoma (HCC). The S-gene with a full size was amplified and made in order. The analysis of a phylogenetic tree showed that genotype D was the most dominant genotype in 
patients. Moreover, this analysis identified two strains with genotype E isolated with active carriers. The detailed phylogenetic analysis confirmed four subgenotypes of HBV D, D1 (93\%, $\mathrm{n}=296)$, D2 (0.02\%, $\mathrm{n}$ $=5)$, D3 $(0.003 \%, \mathrm{n}=1)$, and $\mathrm{D} 4(0.003 \%, \mathrm{n}=1)$. Besides, six strains of genotype $\mathrm{D}$ were not included in the subgenotype of HBV D. The S-gene from eight strains showed a recombination signal between genotype $\mathrm{D}$ and $\mathrm{A}$ and between genotype $\mathrm{D}$ and $\mathrm{E}$. Some strains save an important medical point of mutation at the protein level. Along with the domination of HBV genotype D, the isolation of genotype $\mathrm{E}$ and some recombinant strains of patients from Saudi Arabia is the important result for the decision of involving the therapeutic intervention for patients. The development of vaccines and the detection of mutation passing the diagnostics in the antigenic epitopes at HBsAg will be beneficial for the authority of public health. Furthermore, the diversity at the nucleotide and amino acid levels, as well as the difference in the proportion of $\mathrm{dN} / \mathrm{dS}$ in PreS1, PreS2, and HBsAg showed a tendency of selective pressure of non-active status to advanced liver disease (12).

The School-Based Vaccination (SBV) and the inspection of students' vaccination records at school potentially optimized the coverage of vaccination in school-age children. The main purpose of this paper was to describe the adoption of SBV by some countries from 2008 to 2017, including the targeted age groups and vaccines given in 2017, as reported annually through the Joint Reporting with World Health Organization (WHO)-United Nations children's fund (UNICEF) Forms (JRF). Extending the previous analyses, the rate of elementary school enrollment and the specific Home-Based Recording (HBR) of the country are associated with the data taken from WHO-UNICEF JRF to identify the countries with high potency to perform an inspection of vaccination recording at school. The proportion of countries reporting the vaccination, at least one dose recommended regularly at school environment, increased from 95 (out of 163 reports; 58\%) in 2008 to 108 (from 181 reports; 60\%) in 2017. Other 13 countries reporting to use SBV in 2017 were included in 31 countries with unavailable SBV data from JRF in 2017. The most common antigen sent through SBV in 2017 was tetanus (94 countries), diphtheria (89 countries), and human papillomavirus (52 countries). Among 93 countries with available data for the clean elementary school enrollment and the history of getting HBR, $52(56 \%)$ countries had $\geq 80 \%$ of the clean elementary school enrollment, and $\geq 80 \%$ of children aged $12-23$ months had ever received HBR; $33(63 \%)$ of them used SBV. These 33 countries were the role model countries for introducing the regular check-up for vaccination status when enrolling the school or during elementary school in countries that have not performed the program, including Indonesia. With an increased number of new vaccines and boosters for children targeting schoolage children, the implementation of SBV and the inspection of students' vaccination records at school can help increase the coverage of vaccination; however, additional data are needed to evaluate the global prevalence of the inspection of vaccination status at school and identify the factors facilitating the optimal implementation of this strategy (13).

\section{Conclusion}

The global prevalence of HBsAg in 2016 was 3.9\%. The prevalence of HBsAg in Indonesia in 2016 showed that the population of adult people and pregnant women were at risk of being infected with hepatitis B more than preschool-age and school-age children. Giving hepatitis B vaccine recombinant in adolescents aged 15-18 years is proven to help provide a strong and safe immune along with the decline in anti-HBs in children after universal vaccination for newborn babies. The factors associated with the coverage of immunization completeness and the coverage of hepatitis B birth dose vaccine are as follows: difficulties to visit health centers due to geographic isolation, hepatitis B screening for all pregnant women is not performed as the national program in Indonesia, so the newborn babies born from women with hepatitis B do not receive HBIG in 24 hours, mothers with a firstborn child have 2.84 times higher possibility to vaccinate their children fully than the mothers with $\geq 6$ children, mothers with the lowest economic condition, those giving birth at non-health facilities, and those attending antenatal care less than four times have a lower tendency to vaccinate their children fully. Meanwhile, mothers giving birth assisted by professional birth attendants significantly correlate with the complete immunization status of their children.

According to the national immunization policy in Indonesia, the hepatitis B birth dose vaccine shall be given in 7 days after birth and followed by three combination vaccines, including diphtheria, pertussis, tetanus, and hepatitis B in 2, 3, and 4 months (DPT-HB3). Both vaccines, hexavalent (DTaP-HB-Hib-IPV) and pentavalent (DTwP-HB-Hib), are effective in stimulating the levels of anti-HBs $\geq 10 \mathrm{mIU} / \mathrm{ml}$, and therefore, both of them can be used as a single formula booster for those aged 18 months to simplify the vaccination. A study on the immunogenicity response of newborn babies after getting the four doses of bivalent oral polio vaccine (bOPV), given along with Pentabio and one dose of IPV in the fourth visit is immunogenic and well-tolerated without any serious problem related to the side effects of the vaccine. A previous study identified four genotypes (A, B, C, and D) in adult people in Indonesia; nonetheless, 
genotypes B and C showed the most dominant contribution. Further, four zones of HBV subtypes shared patterns identified in Indonesia: adw, ayw, adr, and mix. The development of vaccines and the detection of mutation passing the diagnostics in the antigenic epitopes in HBsAg will be beneficial for the authority of public health. Further, the diversity at the nucleotide and amino acid levels as well as the difference in the proportion of $\mathrm{dN} / \mathrm{dS}$ in PreS1, PreS2, and HBsAg shows a tendency of selective pressure of non-active status to advanced liver disease. A new policy related to School-Based Vaccination needs to be introduced in Indonesia with a regular medical check-up for vaccination status when enrolling the school or during elementary school. With an increased number of new vaccines and the dose of vaccine booster for children targeting school-age students, SBV implementation, and the inspection of student vaccination record at school can help increase the vaccination coverage; nevertheless, additional data are required to evaluate the global prevalence of inspecting the vaccination status at school and identify the factors facilitating the optimal implementation of this strategy.

\section{References}

1. Sarin SK, Kumar M, Eslam M. Liver diseases in the Asia-Pacific region: a Lancet Gastroenterology \& Hepatology Commission. Lancet Gastroenterol Hepatol [Internet]. 2020;5(2):167-228. Available from: https://www.sciencedirect.com/science/article/abs/pii/S2468125319303425

2. Singh PK. Towards ending viral hepatitis as a public health threat: Translating new momentum into concrete results in South-East Asia. Gut Pathog [Internet]. 2018;10(1):1-5. Available from: https://doi.org/10.1186/s13099-018-0237-X

3. Purwono PB, Juniastuti, Amin M, Bramanthi R, Nursidah, Resi EM, et al. Hepatitis B virus infection in Indonesia 15 years after adoption of a universal infant vaccination program: Possible impacts of low birth dose coverage and a vaccine-escape mutant. Am J Trop Med Hyg. 2016;95(3):674-9.

4. $\quad$ RI K. Hasil Utama Riskesdas Tahun 2018. 2018;

5. Shearer DR, Gamkrelidze I, Nguyen MH, Chen D-S. Global prevalence, treatment, and prevention of hepatitis B virus infection in 2016: a modelling study. Lancet Gastroenterol Hepatol [Internet]. 2018;3(6):383-403. Available https://www.sciencedirect.com/science/article/abs/pii/S2468125318300566\#ceab10

6. Purwono PB, Lusida MI. Studi Hepatitis B Pada Anak-Anak Yang Lahir Di Era. 2012;93-7.

7. Putera EM, Marcia D, Firdarini I, Amin M, Juniastuti J, Purwono PB, et al. Hepatitis B Serology Profiles on Children Aged 1-13 Years Old in Sumenep, Madura. Indones J Trop Infect Dis. 2016;3(2):61.

8. Efendi F, Pradiptasiwi DR, Krisnana I, Kusumaningrum T. Factors associated with complete immunizations coverage among Indonesian children aged 12-23 months. Child Youth Serv Rev [Internet]. 2020;108:104651. Available from: https://www.sciencedirect.com/science/article/abs/pii/S019074091931165X

9. Fadlyana E, Rusmil K, Bachtiar NS. Kekebalan dan Keamanan setelah Mendapat Imunisasi Hepatitis B Rekombinan pada Anak Remaja. Sari Pediatr. 2016;15(2):87.

10. Posuwan N, Wanlapakorn N, Vongpunsawad S. Comparison of hepatitis B surface antibody levels induced by the pentavalent DTwP-HB-Hib versus the hexavalent DTaP-HB-Hib-IPV vaccine, administered to infants at 2, 4, 6, and 18 months of age, following monovalent hepatitis B vaccination at birth. Vaccine [Internet]. 2020;38(7):1643-51. Available from: https://www.sciencedirect.com/science/article/pii/S0264410X19317293

11. Fadlyana E, Dhamayanti M, Tarigan R, Mulia Sari R, Sjafri Bachtiar N, Kartasasmita CB, et al. Immunogenicity and safety profile of a primary dose of bivalent oral polio vaccine given simultaneously with DTwP-Hb-Hib and inactivated poliovirus vaccine at the 4th visit in Indonesian infants. Vaccine [Internet]. 2020;38(8):1962-7. Available from: https://doi.org/10.1016/j.vaccine.2020.01.007

12. Al-Qahtani AA, Pourkarim MR, Trovão NS, Vergote V, Li G, Thijssen M, et al. Molecular epidemiology, phylogenetic analysis and genotype distribution of hepatitis B virus in Saudi Arabia: Predominance of genotype D1. Infect Genet Evol [Internet]. 2020;77(August 2019):104051. Available from: https://doi.org/10.1016/j.meegid.2019.104051

13. R.Feldstein L, Fox G, Shefer A, M.Conklin L, Ward K. School-based delivery of routinely recommended vaccines and opportunities to check vaccination status at school, a global summary, 2008-2017. Vaccine [Internet]. 2020;38(3):680-9. Available from: https://www.sciencedirect.com/science/article/pii/S0264410X19314343 
